\title{
A Trivial Yet Optimal Solution to Vertex Fault Tolerant Spanners
}

\author{
Greg Bodwin* and Shyamal Patel ${ }^{\dagger}$ \\ Georgia Tech
}

\begin{abstract}
We give a short and easy upper bound on the worst-case size of fault tolerant spanners, which improves on all prior work and is fully optimal at least in the setting of vertex faults.
\end{abstract}

\section{Introduction}

This paper concerns spanners, a fundamental primitive used in graph sketching.

Definition 1 (Spanners). A $k$-spanner of a graph $G=(V, E, w)$ is a subgraph $H=\left(V, E^{\prime} \subseteq E, w\right)$ for which $\operatorname{dist}_{H}(s, t) \leq k \cdot \operatorname{dist}_{G}(s, t)$ for all $s, t \in V$.

Spanners have been intensively studied since the mid-80s [28, 27, 4, 3, 18, 19, 29, 6, 11, 1, 21, 2, A staple in the literature is the greedy construction algorithm of Althöfer et al 4, which works as follows: initialize $H$ to be an empty graph, and then for each edge $(u, v)$ in the input graph $G$ in order of increasing weight, add $(u, v)$ to $H$ if currently $\operatorname{dist}_{H}(u, v)>k w_{(u, v)}$. Besides its simplicity and obvious correctness, the greedy algorithm is popular because there is a simple proof that it is existentially optimal 4, 21, meaning that the number of edges in $H$ never exceeds the worst-case number of edges needed for a $k$-spanner over all possible input graphs on as many nodes as $G$.

In practice, spanners are often applied to systems whose parts are prone to sporadic failures. A spanner for such a system must be robust to these failures, giving rise to the notion of fault tolerance:

Definition 2 (Fault Tolerant Spanners). Given a graph $G$, a subgraph $H$ is an $f$ Vertex Fault Tolerant (VFT), resp. Edge Fault Tolerant (EFT), $k$-spanner of $G$ if for any set of $F$ vertices (resp. edges) in $G$ of size $|F| \leq f, H \backslash F$ is a k-spanner of $G \backslash F$.

To construct FT spanners, it is natural to consider the obvious adaptation of the greedy algorithm:

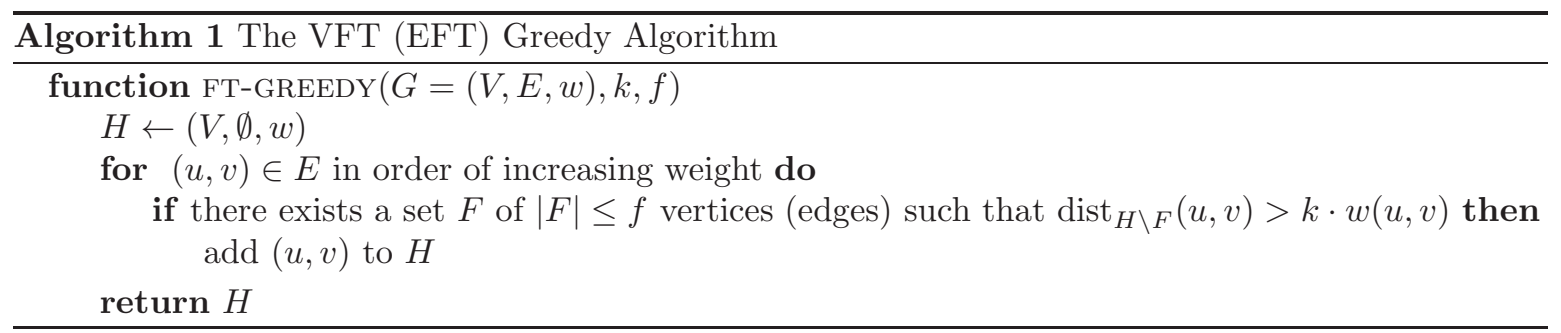

Correctness is again obvious, but unfortunately the analyses used in the non-faulty setting all seem to break for the FT greedy algorithm. Most prior work on FT spanners has thus abandoned the greedy approach in favor of more involved constructions [23, 14, 13, 16, 5] (see also 24, 25, 26, 10, 12, 15, 7, 22, 30, 17, 8); an analysis of the FT greedy algorithm was only obtained recently via fairly complex arguments [9]. In contrast, our main result is a simple analysis of the FT greedy algorithm which improves on all of these previous bounds.

*gbodwin6@cc.gatech.edu

†shyamalpatelb@gmail.com 
Theorem 1 (Main Result). Let $b(n, k)$ be the maximum possible number of edges in a graph on $n$ nodes and girth $>k$. Then any graph $H$ on $n$ nodes returned by the VFT or EFT Greedy Algorithm with parameters $f, k$ satisfies

$$
|E(H)|=O\left(f^{2} \cdot b\left(\frac{n}{f}, k+1\right)\right) .
$$

This upper bound is best possible in the VFT setting, for any construction algorithm, due to a simple lower bound construction in [9] (meaning that any asymptotic tradeoff between $n, f, k,|E(H)|$ not promised by this theorem does not exist in general). In the EFT setting, the bound of Theorem 1 was already known to be the best possible tradeoff when $k<5[9$, but for larger $k$ it is still conceivable to improve the upper bound as far as

$$
|E(H)| \stackrel{?}{=} O\left(f \cdot b\left(\frac{n}{\sqrt{f}}, k+1\right)+n f\right) .
$$

It remains a major open question to asymptotically determine $b(n, k)$; the only known upper bound is the folklore Moore bounds which state $b(n, k)=O\left(n^{1+1 /\lfloor k / 2\rfloor}\right)$. Plugging this into Theorem 1 yields:

Corollary 2. For any graph $H$ on $n$ nodes returned by the VFT or EFT Greedy Algorithm with parameters $f, 2 k-1$, we have

$$
|E(H)|=O\left(n^{1+1 / k} f^{1-1 / k}\right) .
$$

This corollary improves over the previous best upper bound in 9 by a factor of $\exp (k)$. The famous Erdös girth conjecture 20] posits that the Moore Bounds are tight, which would then imply that this corollary is best possible, at least for VFT spanners.

An open question left by this work is to improve the runtime of the FT greedy algorithm: in a naive implementation it is exponential in $f$. It would be interesting to improve this dependence, or perhaps to find a different fast algorithm achieving the existential size bounds proved in this paper. We note that [16] gives a construction with polynomial runtime dependence on $f$, at the price of somewhat suboptimal spanner size.

\section{Proof of Main Result}

We will state the proof in the VFT setting here; the proof in the EFT setting is essentially identical.

Definition 3 (Blocking Set). Given a graph $G=(V, E)$, a $k$-blocking set for $G$ is a set $B \subseteq V \times E$ such that (1) every $(v, e) \in B$ has $v \notin e$, and (2) for every cycle $C$ in $G$ on $\leq k$ edges, there exists $(v, e) \in B$ such that $v, e \in C$.

Lemma 3. Any graph $H$ returned by the VFT greedy algorithm with parameters $k, f$ has a $(k+1)$ blocking set of size at most $f|E(H)|$.

Proof. For each edge $e=(u, v) \in H$, let $F_{e}$ be the set of nodes such that $\operatorname{dist}_{H \backslash F_{e}}(u, v)>k w_{e}$ when $e$ is added to $H$. Let

$$
B:=\left\{(x, e) \mid e \in E(H), x \in F_{e}\right\} ;
$$

since $\left|F_{e}\right| \leq f$ for all $e$, we have $|B| \leq f|E(H)|$. We now show that $B$ is a $(k+1)$-blocking set. Let $C$ be any cycle on $\leq k+1$ edges in the final graph $H$ and let $e=(u, v)$ be the last edge in $C$ considered by the greedy algorithm. By construction there is a $u \rightsquigarrow v$ path (through $C$ ) of total weight $\leq k w_{(u, v)}$ when $e$ is added to $H$, and so some node $x \in C \backslash\{u, v\}$ must be included in $F_{e}$. Thus $(x, e) \in B$.

Lemma 4. Let $H$ be any graph on $n$ nodes and $m$ edges, let $f=o(n)$ be a parameter, and suppose $H$ has a $(k+1)$-blocking set $B$ of size $|B| \leq f|E(H)|$. Then $H$ has a subgraph on $O(n / f)$ nodes, $\Omega\left(m / f^{2}\right)$ edges, and girth $>k+1$.

Proof. Let $H^{\prime}$ be an induced subgraph of $H$ on a uniformly random subset of exactly $\lceil n /(2 f)\rceil$ vertices, let $B^{\prime}:=B \cap\left(E\left(H^{\prime}\right) \times V\left(H^{\prime}\right)\right)$, and let $H^{\prime \prime}$ be obtained from $H^{\prime}$ by deleting every edge contained in any pair in $B^{\prime}$. The graph $H^{\prime \prime}$ has girth $>k+1$, since by definition of blocking sets we have now deleted at least one node or edge from every cycle in $H$ on $\leq k+1$ edges. Additionally: 
- Each edge in $E(H)$ survives in $E\left(H^{\prime}\right)$ iff both of its endpoints survive in $V\left(H^{\prime}\right)$, which happens with probability

$$
\frac{\lceil n /(2 f)\rceil}{n} \cdot \frac{\lceil n /(2 f)\rceil-1}{n-1}=(1+o(1)) \frac{1}{4 f^{2}} .
$$

- Each pair $(x,(u, v)) \in B$ survives in $B^{\prime}$ iff all of $x, u, v$ survive in $V\left(H^{\prime}\right)$, which happens with probability

$$
\frac{\lceil n /(2 f)\rceil}{n} \cdot \frac{\lceil n /(2 f)\rceil-1}{n-1} \cdot \frac{\lceil n /(2 f)\rceil-2}{n-2}=(1+o(1)) \frac{1}{8 f^{3}}
$$

We may now compute

$\mathbb{E}\left[\left|E\left(H^{\prime \prime}\right)\right|\right] \geq \mathbb{E}\left[\left|E\left(H^{\prime}\right)\right|-\left|B^{\prime}\right|\right]=(1+o(1))\left(\frac{|E(H)|}{4 f^{2}}-\frac{|B|}{8 f^{3}}\right) \geq(1+o(1))\left(\frac{m}{4 f^{2}}-\frac{m}{8 f^{2}}\right)=\Omega\left(\frac{m}{f^{2}}\right)$.

There exists a possible setting of $H^{\prime \prime}$ which matches or exceeds this expectation, which thus has $\Omega\left(m / f^{2}\right)$ edges and satisfies the lemma.

Proof of Theorem 1. If $f=\Omega(n)$, then Theorem 1 holds trivially since it states $|E(H)|=O\left(n^{2}\right)$. Otherwise, let $H$ be an output graph of the FT Greedy algorithm on $n$ edges and $m$ nodes. By Lemmas 3 and $H$ has a subgraph of girth $>k+1$ on $O(n / f)$ nodes and $\Omega\left(m / f^{2}\right)$ edges, and hence

$$
\begin{aligned}
b(O(n / f), k+1) & =\Omega\left(\frac{m}{f^{2}}\right) \\
m & =O\left(f^{2} \cdot b(n / f, k+1)\right) .
\end{aligned}
$$

We conclude by remarking on a limitation of our approach. Our definition of blocking sets is very VFT-centric; since a gap remains in the EFT setting, it is tempting to try to adapt this definition to the EFT setting in search of better upper bounds. In particular, let us say that an edge $k$-blocking set is a set of distinct edge pairs such that every $(\leq k)$-cycle $C$ has $e_{1}, e_{2} \in C$ for some $\left(e_{1}, e_{2}\right) \in B$. It is easy to show that any graph $H$ returned by the EFT greedy algorithm with parameters $f, k$ admits an edge $(k+1)$-blocking set of size $\leq f|E(H)|$ (the analog of Lemma 3). We would then need an improved analog of Lemma 4 in order to get improved upper bounds on EFT spanners for $k \geq 5$. However, no such improvement is possible: for any $k$ we can show a graph $H$ on $\Omega\left(f^{2} \cdot b(n / f, k+1)\right)$ edges that has an edge $(k+1)$-blocking set of size $\leq f|E(H)|$, and so our analog of Lemma 3 alone is not powerful enough to get improved upper bounds in the EFT setting. Specifically, this $H$ is the same as the VFT lower bound graph of [9]: it is the Cartesian product of an arbitrary graph of girth $>k+1$ with a biclique on $\lfloor f / 2\rfloor$ nodes; the blocking set is then all pairs of edges that share an endpoint in the product graph and which correspond to the same edge in the initial high-girth graph. Hence any improvement to our EFT upper bounds (if possible) will need to exploit stronger properties of $H$ than just the existence of a small edge blocking set.

\section{References}

[1] Abboud, A., And Bodwin, G. The $4 / 3$ additive spanner exponent is tight. In Proceedings of the 48th Annual ACM SIGACT Symposium on Theory of Computing (STOC) (2016), ACM Special Interest Group on Algorithms and Computation Theory, pp. 351-361.

[2] Abboud, A., Bodwin, G., And Pettie, S. A hierarchy of lower bounds for sublinear additive spanners. In Proceedings of the 28th Annual ACM-SIAM Symposium on Discrete Algorithms (SODA) (2017), Society for Industrial and Applied Mathematics, pp. 568-576.

[3] Aingworth, D., Chekuri, C., Indyk, P., And Motwani, R. Fast estimation of diameter and shortest paths (without matrix multiplication). SIAM Journal on Computing 28, 4 (1999), $1167-1181$.

[4] Althöfer, I., Das, G., Dobkin, D., Joseph, D., And Soares, J. On sparse spanners of weighted graphs. Discrete \& Computational Geometry 9, 1 (1993), 81-100. 
[5] Ausiello, G., Franciosa, P. G., Italiano, G. F., and Ribichini, A. Computing graph spanners in small memory: fault-tolerance and streaming. Discrete Mathematics, Algorithms and Applications 2, 04 (2010), 591-605.

[6] Baswana, S., Kavitha, T., Mehlhorn, K., and Pettie, S. Additive spanners and ( $\alpha$, $\beta$ )-spanners. ACM Transactions on Algorithms (TALG) 7, 1 (2010), 5.

[7] Bernstein, A., And Karger, D. Improved distance sensitivity oracles via random sampling. In Proceedings of the nineteenth annual ACM-SIAM symposium on Discrete algorithms (2008), Society for Industrial and Applied Mathematics, pp. 34-43.

[8] Bilo, D., Guala, L., Leucci, S., and Proietti, G. Compact and Fast Sensitivity Oracles for Single-Source Distances. In 24th Annual European Symposium on Algorithms (ESA 2016) (Dagstuhl, Germany, 2016), P. Sankowski and C. Zaroliagis, Eds., vol. 57 of Leibniz International Proceedings in Informatics (LIPIcs), Schloss Dagstuhl-Leibniz-Zentrum fuer Informatik, pp. 13:1$13: 14$.

[9] Bodwin, G., Dinitz, M., Parter, M., and Williams, V. V. Optimal vertex fault tolerant spanners (for fixed stretch). In Proceedings of the 29th Annual ACM-SIAM Symposium on Discrete Algorithms (SODA) (2018), Society for Industrial and Applied Mathematics, pp. 1884-1900.

[10] Bodwin, G., Grandoni, F., Parter, M., and Williams, V. V. Preserving distances in very faulty graphs. In Proceedings of the 44th International Colloquium on Automata, Languages, and Programming (ICALP) (2017), European Association for Theoretical Computer Science, pp. 73:1$73: 14$.

[11] Chechik, S. New additive spanners. In Proceedings of the 24th Annual ACM-SIAM Symposium on Discrete Algorithms (SODA) (2013), SIAM, pp. 498-512.

[12] Chechik, S., Cohen, S., Fiat, A., And Kaplan, H. $(1+\varepsilon)$-approximate f-sensitive distance oracles. In Proceedings of the Twenty-Eighth Annual ACM-SIAM Symposium on Discrete Algorithms (2017), Society for Industrial and Applied Mathematics, pp. 1479-1496.

[13] Chechik, S., Langberg, M., Peleg, D., And Roditty, L. Fault tolerant spanners for general graphs. SIAM Journal on Computing 39, 7 (2010), 3403-3423.

[14] Czumaj, A., And Zhao, H. Fault-tolerant geometric spanners. Discrete \& Computational Geometry 32, 2 (2004), 207-230.

[15] Demetrescu, C., Thorup, M., Chowdhury, R. A., and Ramachandran, V. Oracles for distances avoiding a failed node or link. SIAM Journal on Computing 37, 5 (2008), 1299-1318.

[16] Dinitz, M., And Krauthgamer, R. Fault-tolerant spanners: better and simpler. In Proceedings of the 30th annual ACM SIGACT-SIGOPS symposium on Principles of distributed computing (2011), ACM, pp. 169-178.

[17] Dinitz, M., And Zhang, Z. Approximating low-stretch spanners. In Proceedings of the twentyseventh annual ACM-SIAM symposium on Discrete algorithms (2016), Society for Industrial and Applied Mathematics, pp. 821-840.

[18] Dor, D., Halperin, S., And Zwick, U. All-pairs almost shortest paths. Siam Journal on Computing (SICOMP) 29, 5 (2000), 1740-1759.

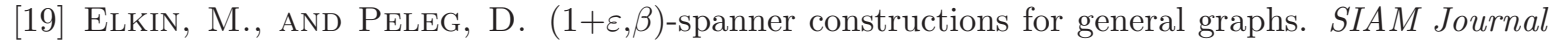
on Computing 33, 3 (2004), 608-631.

[20] Erdős, P. Extremal problems in graph theory. In Proceedings of the Symposium on Theory of Graphs and its Applications (1963), p. 2936.

[21] Filtser, A., And Solomon, S. The greedy spanner is existentially optimal. In Proceedings of the 2016 ACM Symposium on Principles of Distributed Computing (2016), ACM, pp. 9-17. 
[22] Grandoni, F., and Williams, V. V. Improved distance sensitivity oracles via fast singlesource replacement paths. In Foundations of Computer Science (FOCS), 2012 IEEE 53rd Annual Symposium on (2012), IEEE, pp. 748-757.

[23] Levcopoulos, C., Narasimhan, G., And Smid, M. Efficient algorithms for constructing faulttolerant geometric spanners. In Proceedings of the thirtieth annual ACM symposium on Theory of computing (1998), ACM, pp. 186-195.

[24] Parter, M. Dual failure resilient bfs structure. In Proceedings of the 2015 ACM Symposium on Principles of Distributed Computing (2015), ACM, pp. 481-490.

[25] Parter, M., And Peleg, D. Sparse fault-tolerant bfs trees. In European Symposium on Algorithms (2013), Springer, pp. 779-790.

[26] Parter, M., And Peleg, D. Fault-tolerant approximate bfs structures. ACM Transactions on Algorithms (TALG) 14, 1 (2018), 10.

[27] Peleg, D., And Ullman, J. An optimal synchronizer for the hypercube. SIAM Journal on Computing (SICOMP) 18, 4 (1989), 740-747.

[28] Peleg, D., and Upfal, E. A trade-off between space and efficiency for routing tables. Journal of the ACM (JACM) 36, 3 (1989), 510-530.

[29] Thorup, M., And Zwick, U. Spanners and emulators with sublinear distance errors. In Proceedings of the 17th Annual ACM-SIAM Symposium on Discrete Algorithms (SODA) (2006), Society for Industrial and Applied Mathematics, pp. 802-809.

[30] Weimann, O., And Yuster, R. Replacement paths and distance sensitivity oracles via fast matrix multiplication. ACM Transactions on Algorithms (TALG) 9, 2 (2013), 14. 